\title{
Fractionation of Poplar Wood using a Bifunctional Aromatic Acid under Mild
}

\section{Conditions}

Duo Hea ${ }^{\mathrm{a}}$, Jingshun Zhuang ${ }^{\mathrm{b}}$, Yan Jiang ${ }^{\mathrm{c}}$, Danni Xie ${ }^{\mathrm{a}}$, Chang Geun Yoo ${ }^{\mathrm{b} *}$, and Qiang Yang*

aDepartment of Chemical and Paper Engineering, College of Applied Science and Engineering, Western Michigan University, Kalamazoo, MI 49008, USA.

${ }^{b}$ Department of Chemical Engineering, College of Environmental Science and Forestry, State University of New York, Syracuse, NY, USA.

${ }^{c}$ College of Light Industry and Food Engineering, Guangxi University, Nanning, 530004, China.

* To whom correspondence should be addressed. E-mail address: qiang.yang@wmich.edu, cyoo05@esf.edu.

SUPPLEMENTARY INFORMATION (PAGES S1-24)

CONTENTS (contains 3 tables, 18 figures, and references)

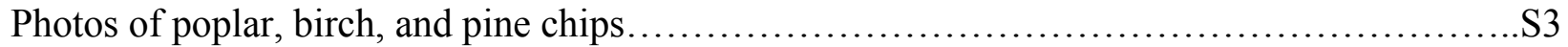

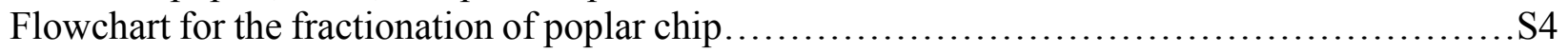

The disintegration of softened poplar chips using a glass rod............................... S5

The calculated $\log$ D value and sigma potential profile of 4-Cl-BSA............................ 6

Chemical structures of lignin monomers, dimers, and trimers.............................. 7

The calculated $\log \mathrm{D}$ values, sigma profiles and sigma potentials for lignin monomers, dimers, and

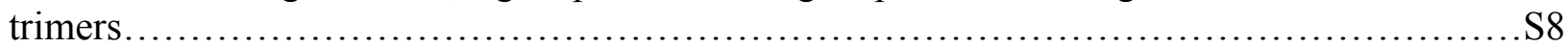

Comparison in solubilizing lignin by 4-Cl-BSA, acetone, ethanol, and tetrahydrofuran............S9

Effects of 4-Cl-BSA concentration and 4-Cl-BSA/polar ratio on the lignin yield................S10

Photos of the dissolved lignin and deposited lignin in water................................... 11

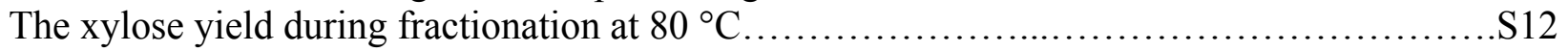




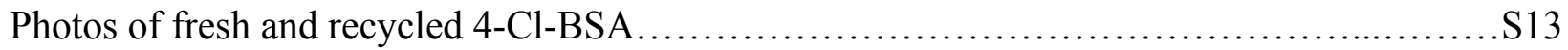

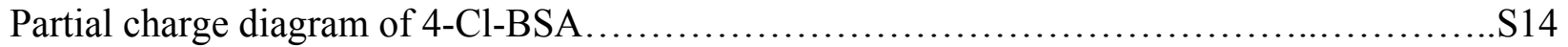

Schematic illustration of the reactions of 4-Cl-BSA with lignin.............................. 15

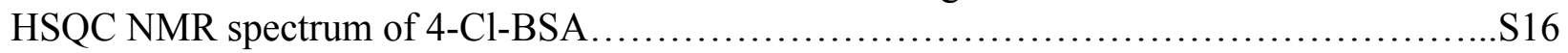

XRD spectra of cellulose fibres isolated from different conditions.........................S17

Degrees of polymerization for cellulose fibres isolated from different conditions...............S18

Chemical structures of 4-Cl-BSA, di-Cl-BSA, 4-Br-BSA, TsOH, PSA, and BSA...............S19

The calculated log D values, sigma profiles, and sigma potential profiles for di-Cl-BSA, 4-Cl-

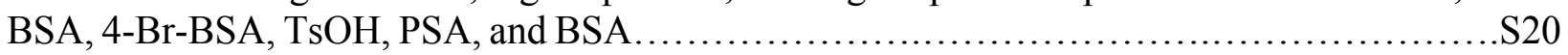

Compositions and inter-unit linkage contents of BSA-fractionated poplar lignin samples.......S21

Molecular weights of BSA-fractionated poplar lignin samples..............................S22

The lignin samples from poplar chips fractionated by 4-Cl-BSA and the reported batch and flow-

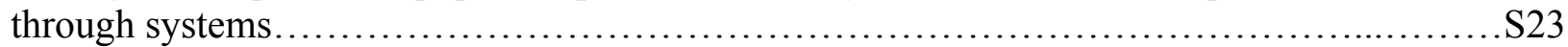

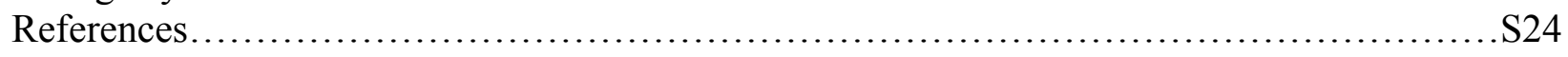



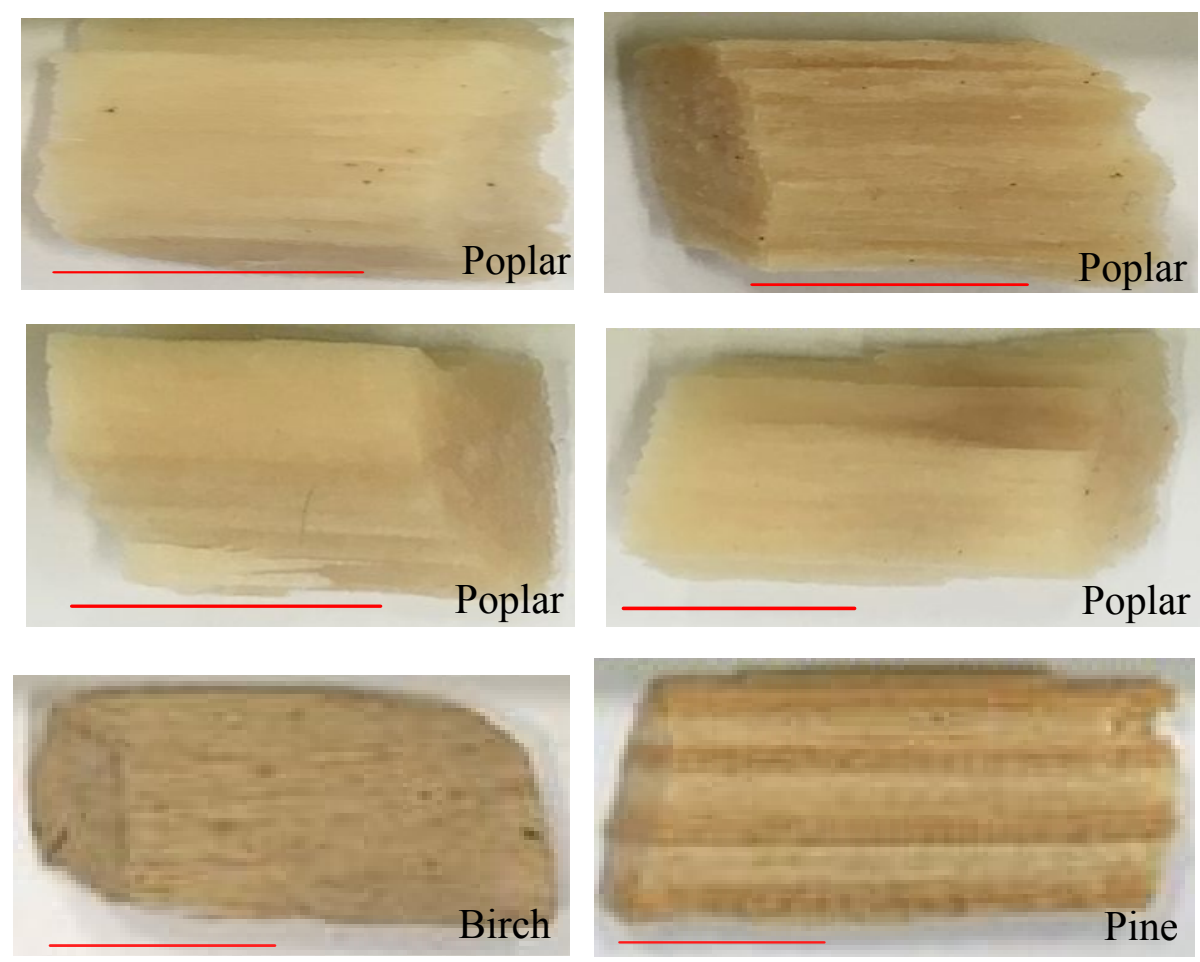

Figure S1. Photos of poplar, birch, and pine chips (scale bar is $1 \mathrm{~cm}$ ). 


\section{Poplar Chip}

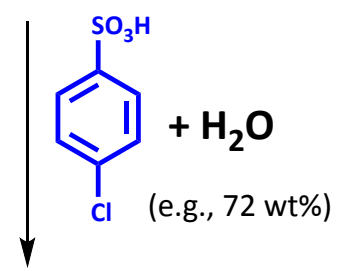

\section{Fractionation/Disintegration}

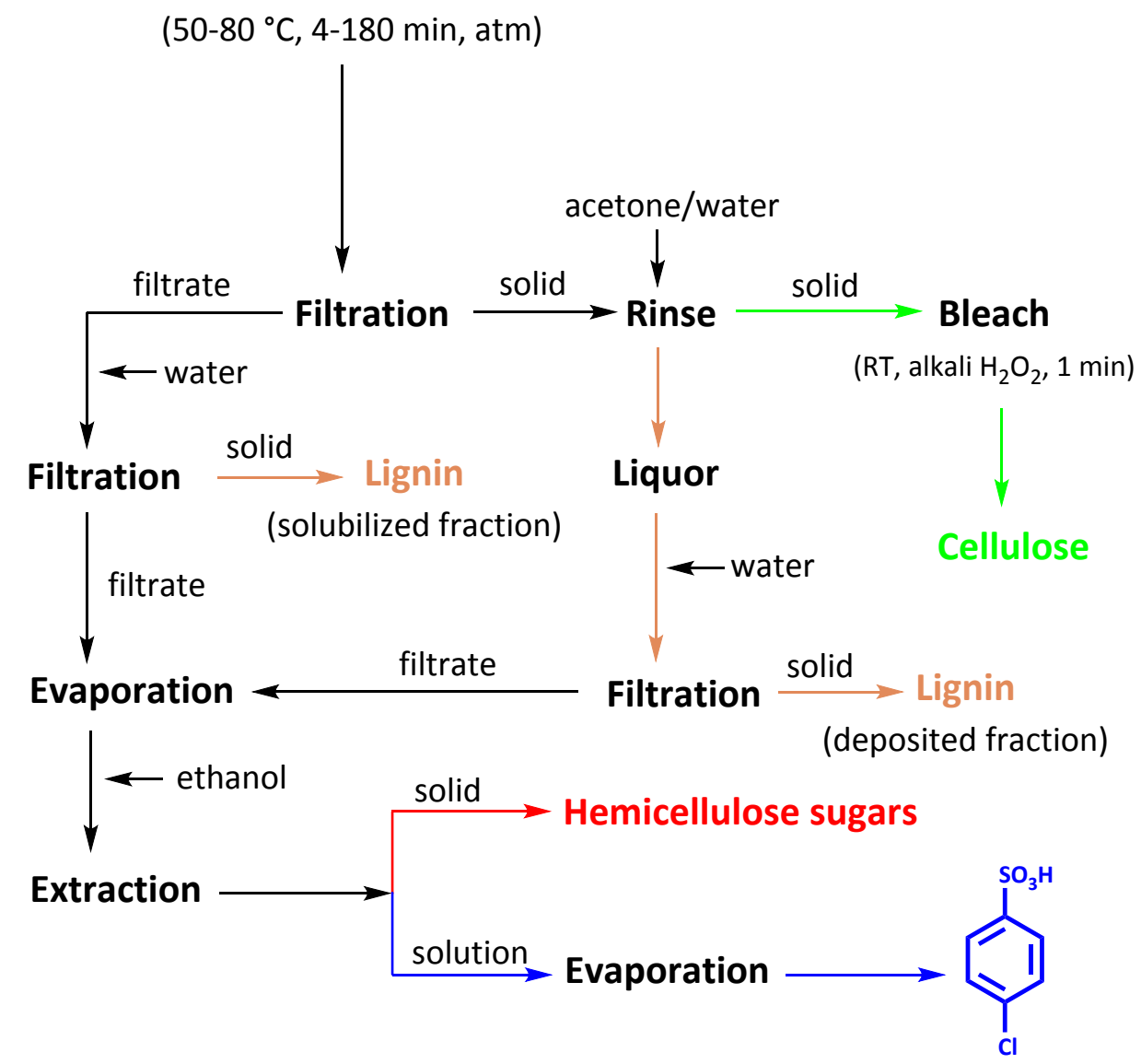

Figure S2. Flowchart for the fractionation of poplar chip by the aqueous $72 \% 4-\mathrm{Cl}-\mathrm{BSA}$ solution. 


\section{A: 0.4 g poplar chip}
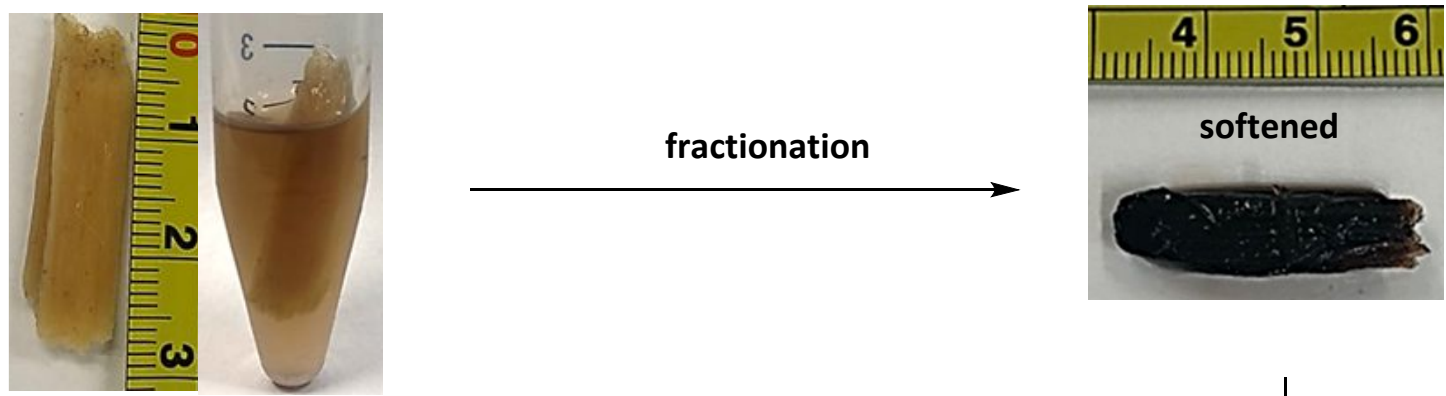

disintegration

pressure:

$140 \mathrm{~g} / \mathrm{cm}^{2}$
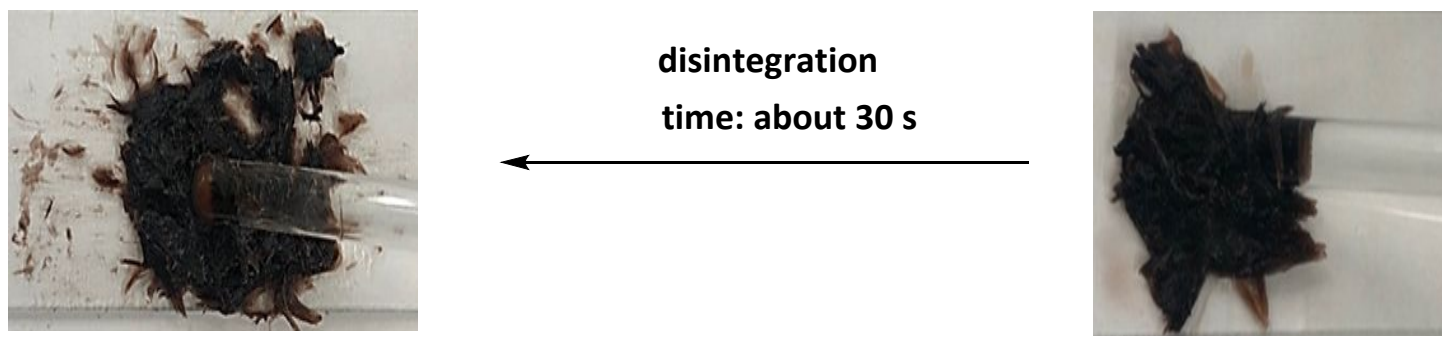

\section{B: 3 g poplar chips}

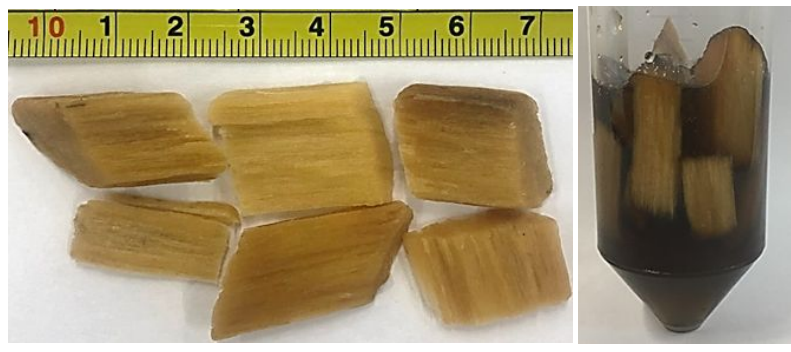

(1) fractionation

(2) disintegration about $1 \mathrm{~min}$

Figure S3. The disintegration of softened poplar chips using a glass rod. 

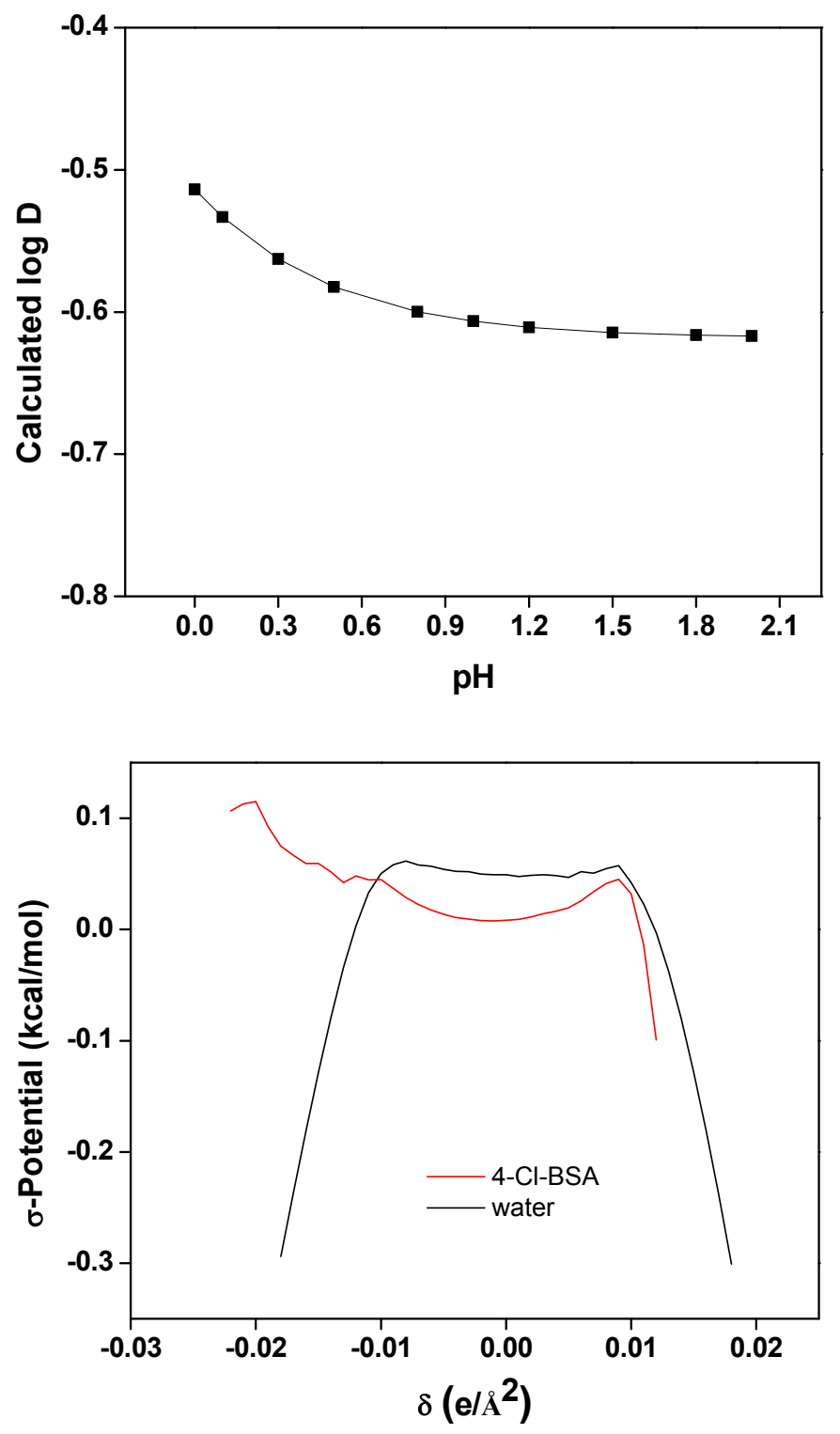

Figure S4. The calculated $\log$ D value and sigma potential profile of 4-Cl-BSA. 

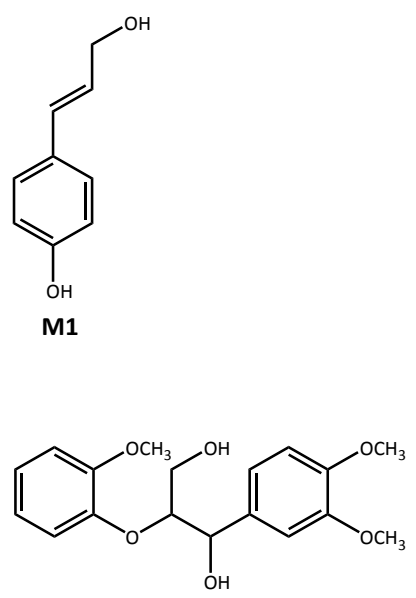

D1

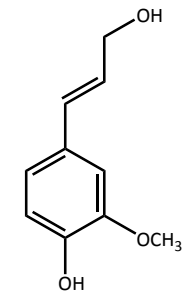

M2

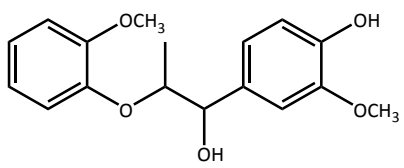

D2
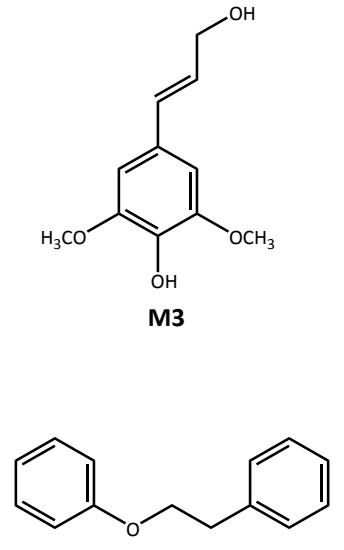

D3<smiles>COc1ccc(C(O)C(CO)Oc2ccc(C(O)C(CO)Oc3ccccc3O)cc2O)cc1O</smiles>

$\mathrm{T} 1$<smiles>COc1cc(C(O)C(CO)Oc2ccccc2O)ccc1Oc1ccc(C(O)C(CO)Oc2ccccc2O)c(O)c1O</smiles>

T2<smiles>COc1ccc(Oc2cc(C3OCC4C(c5ccc(OC)c(OC)c5)OCC34)cc(OC)c2OC)c(O)c1</smiles>

Figure S5. Chemical structures of lignin monomers (M1-M3), dimers (D1-D3), and trimers (T1T3). 

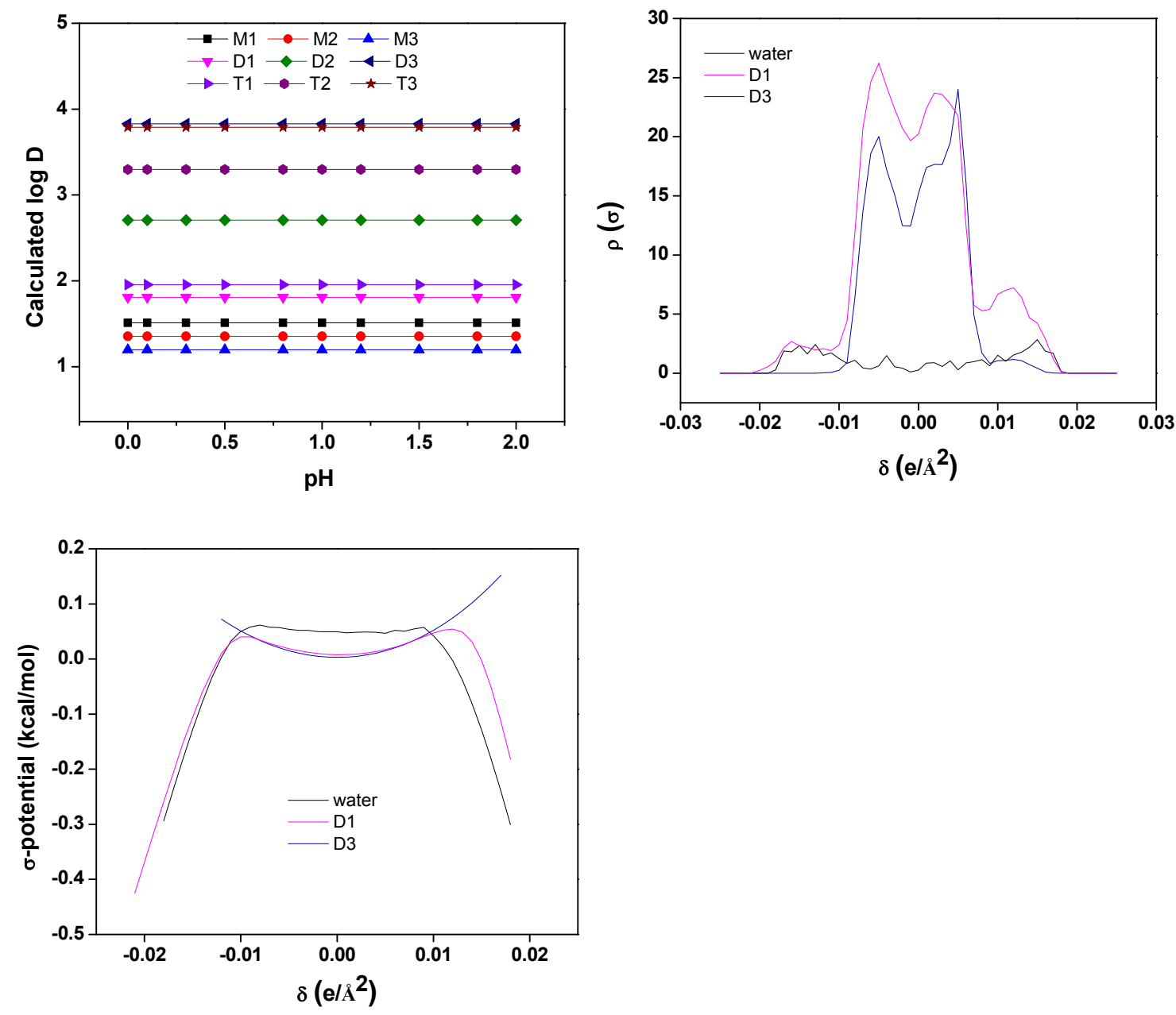

Figure S6. The calculated log D values, sigma profiles and sigma potentials for lignin monomers (M1-M3), dimers (D1-D3), and trimers (T1-T3). 


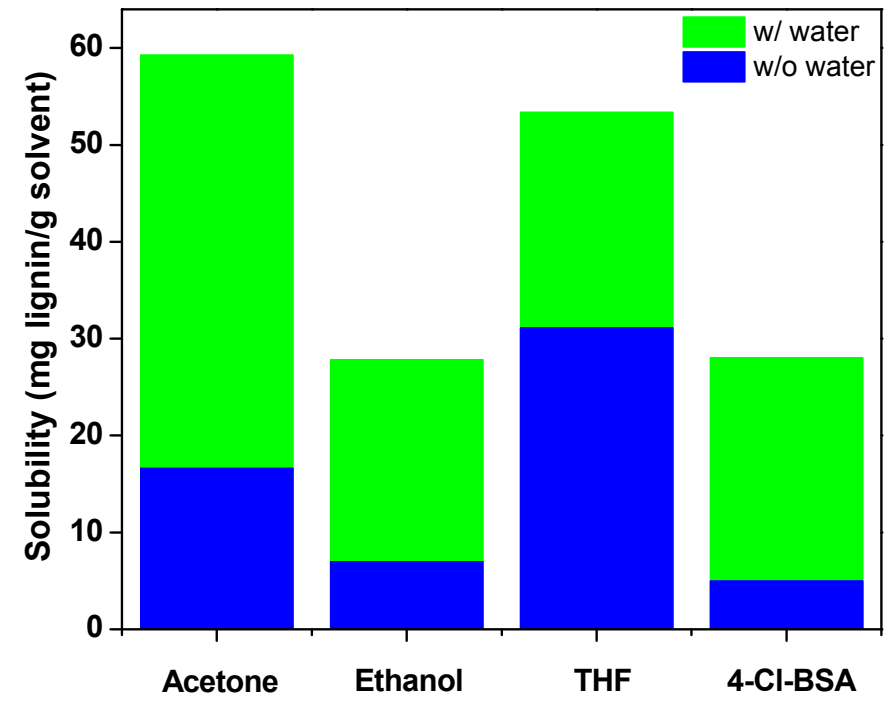

Figure S7. Comparison in solubilizing lignin by 4-Cl-BSA, acetone, ethanol, and tetrahydrofuran (THF) without/with the same amount (28\%) of water. 

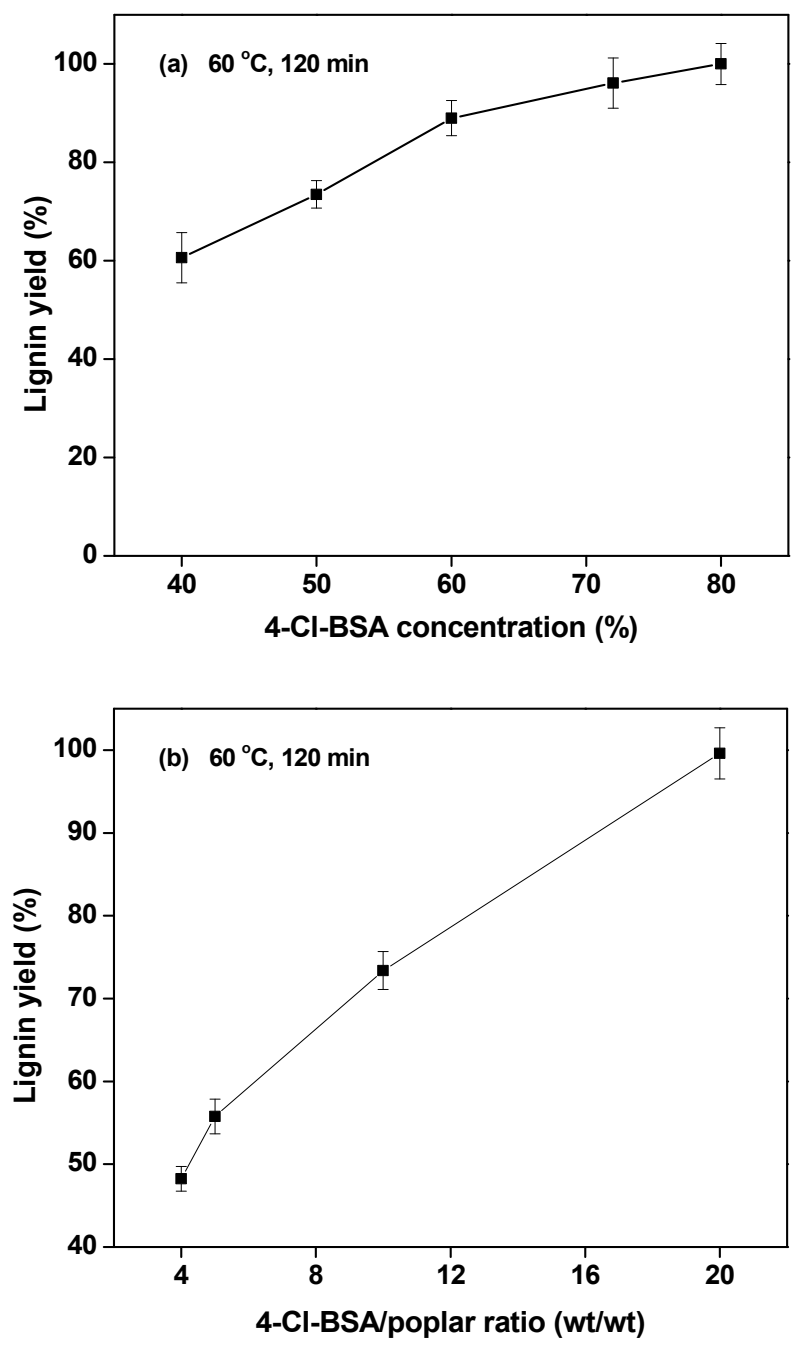

Figure S8. Effects of 4-Cl-BSA concentration (a) and 4-Cl-BSA/polar ratio (b) on the lignin yield. 

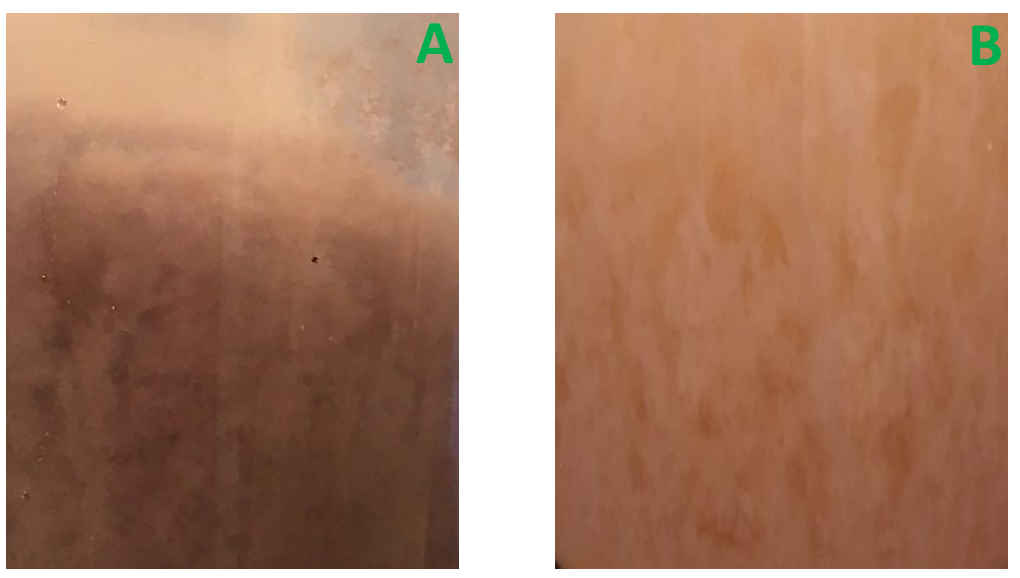

Figure S9. Photos of the dissolved lignin (A) and deposited lignin (B) from the fractionation of poplar chips at $60^{\circ} \mathrm{C}$ for $2 \mathrm{~h}$. 


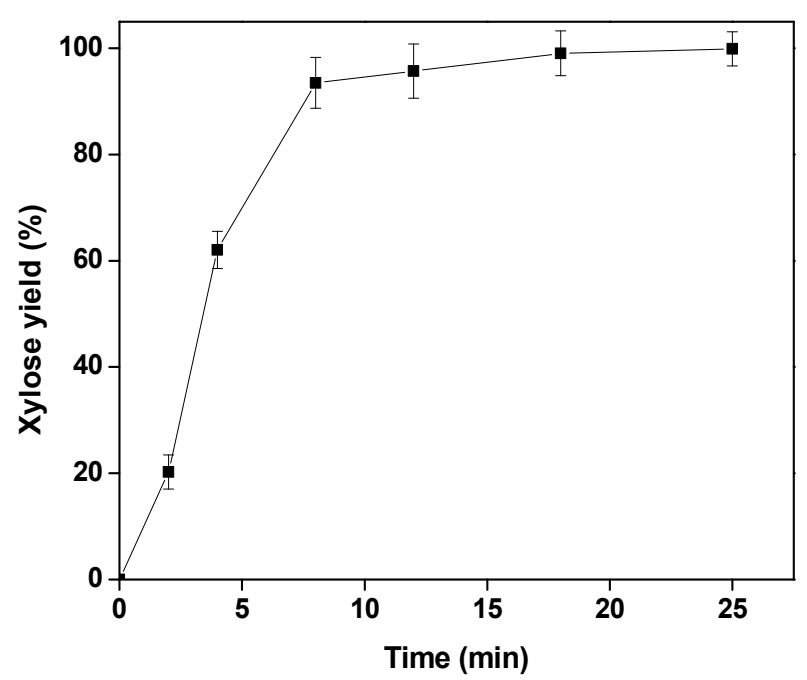

Figure S10. The xylose yield during fractionation at $80^{\circ} \mathrm{C}$. Other hemicellulose sugars (arabinose, mannose and galactose) are negligible. 

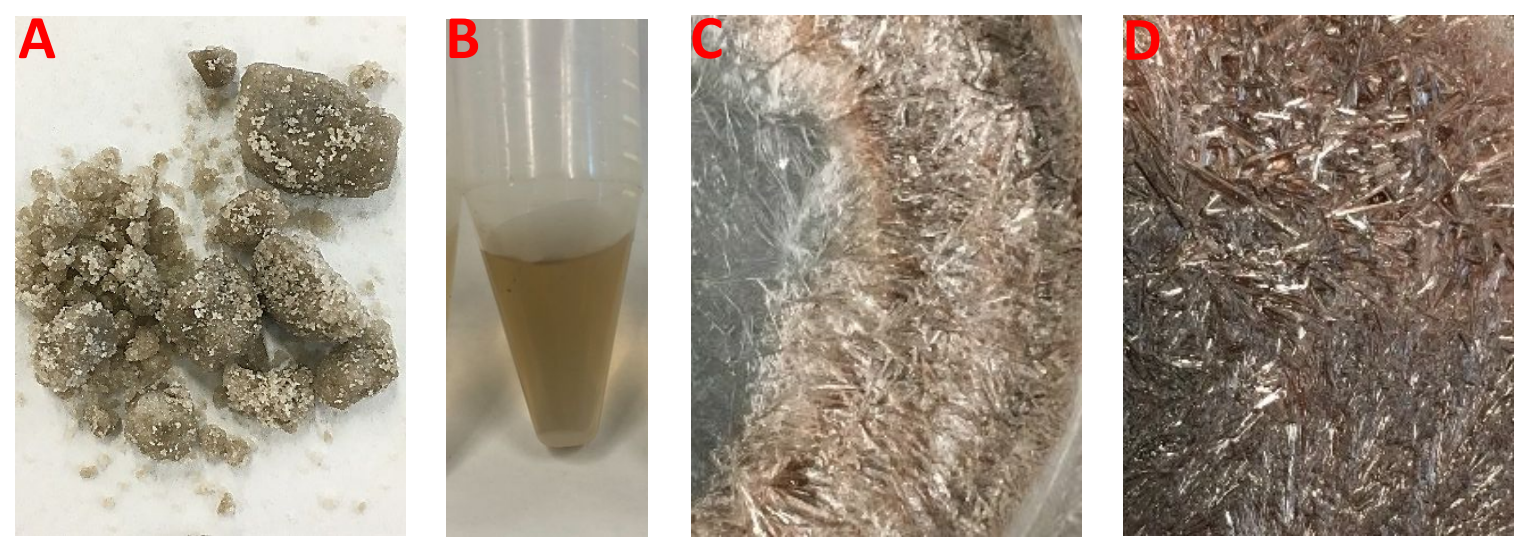

Figure S11. Photos of fresh (A: solid and B: solution) and recycled (C and D) 4-Cl-BSA. 


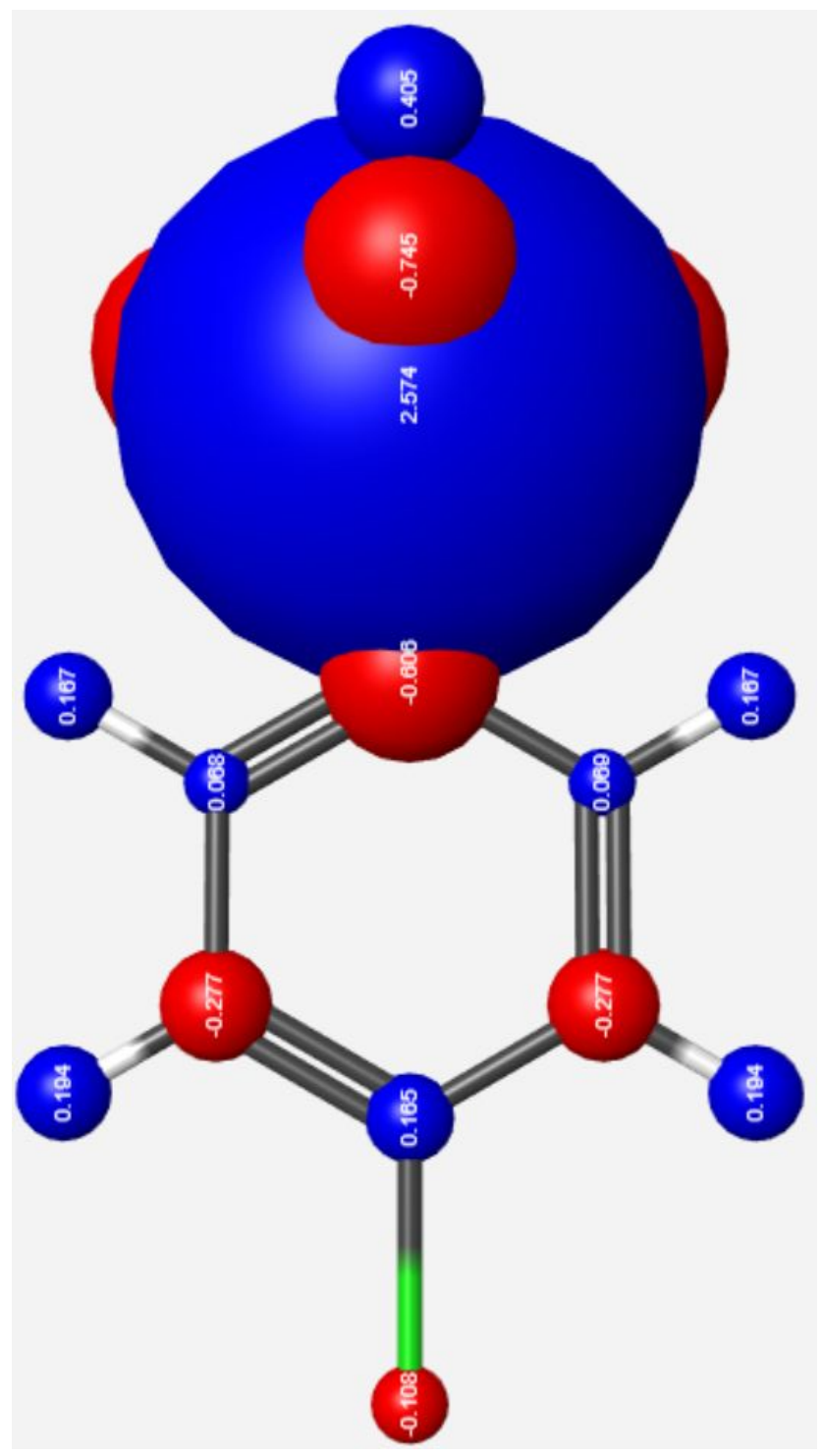

Figure S12. Partial charge diagram of 4-Cl-BSA. 

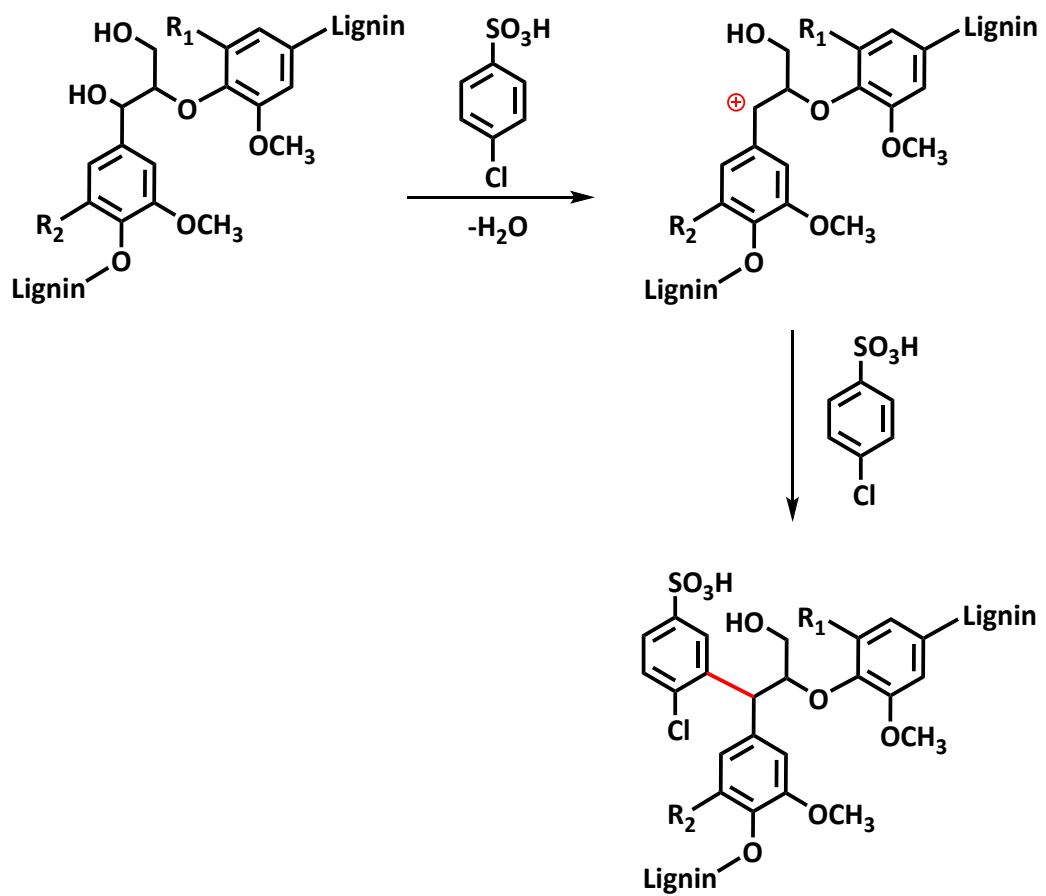

Figure S13. Schematic illustration of the reactions of 4-Cl-BSA with lignin. 


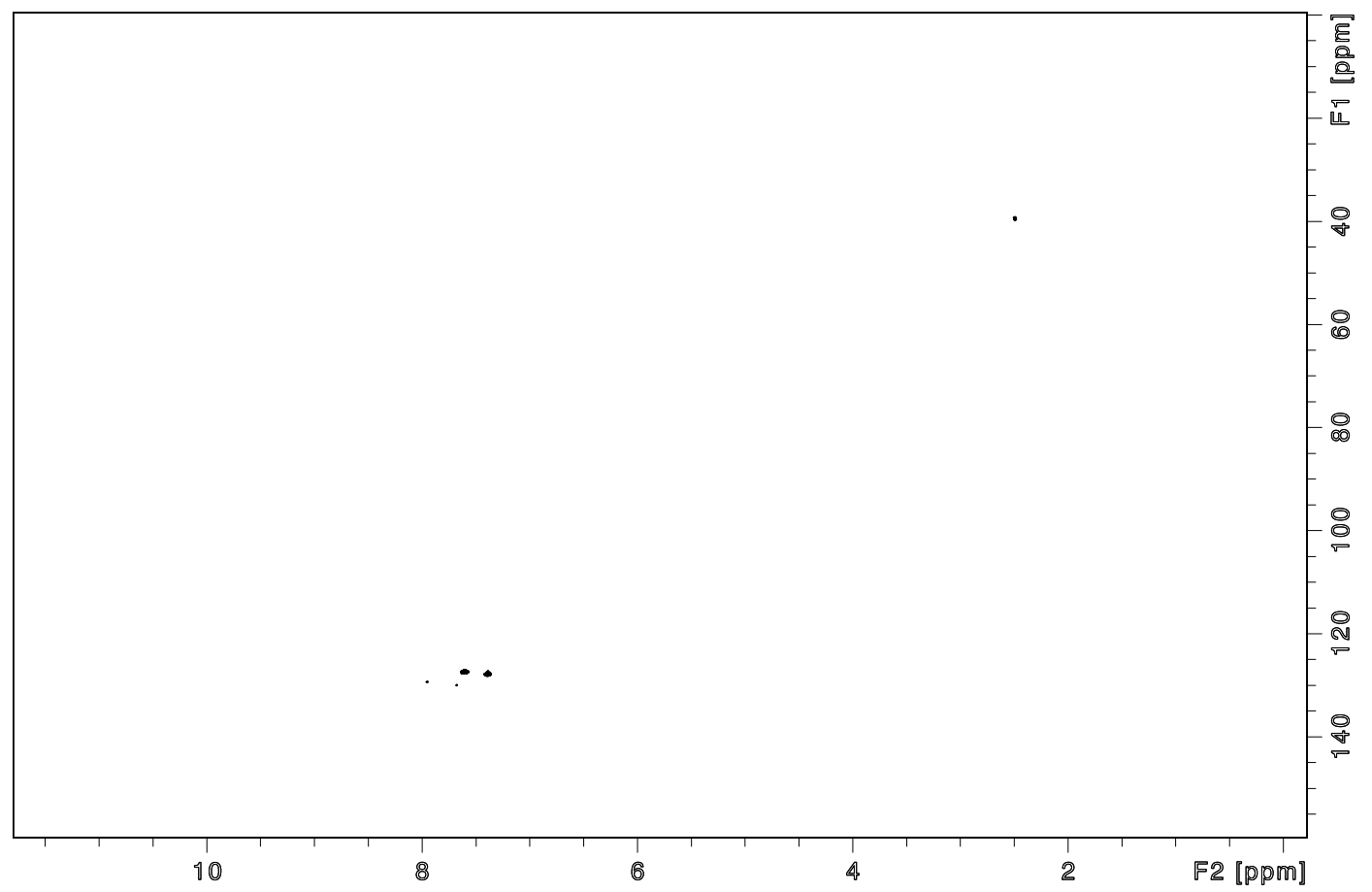

Figure S14. HSQC NMR spectrum of 4-Cl-BSA. 


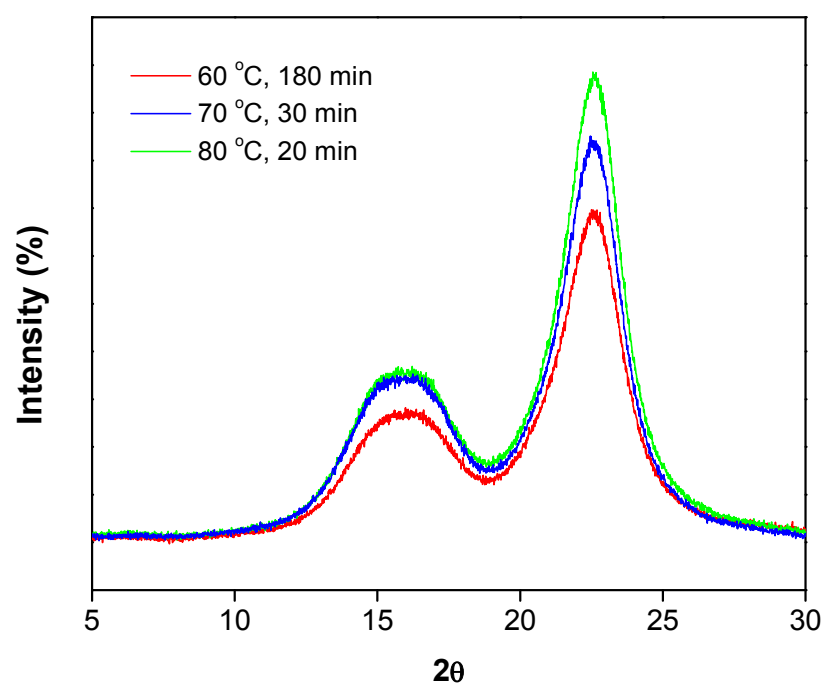

Figure S15. XRD spectra of cellulose fibres isolated from different conditions. 


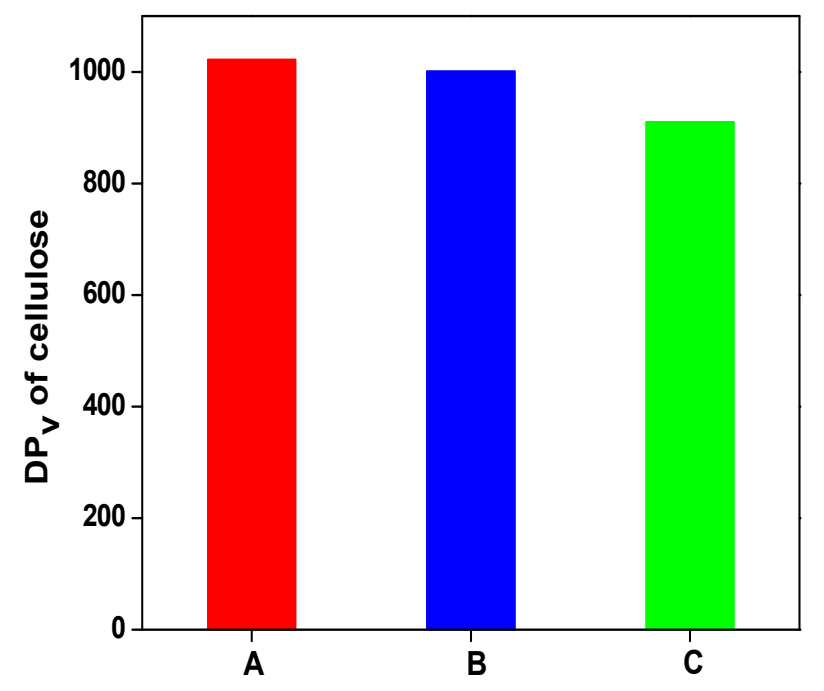

Figure S16. Degrees of polymerization for cellulose fibres isolated from different conditions (A: $60{ }^{\circ} \mathrm{C}, 180 \mathrm{~min}$; $\left.\mathrm{B}: 70^{\circ} \mathrm{C}, 30 \mathrm{~min} ; \mathrm{C}: 80^{\circ} \mathrm{C}, 20 \mathrm{~min}\right)$. 

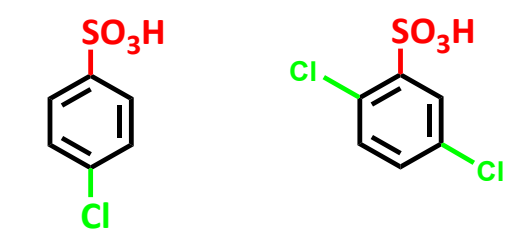

(4-Cl-BSA)

(di-Cl-BSA)

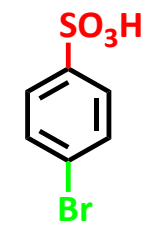

(4-Br-BSA)
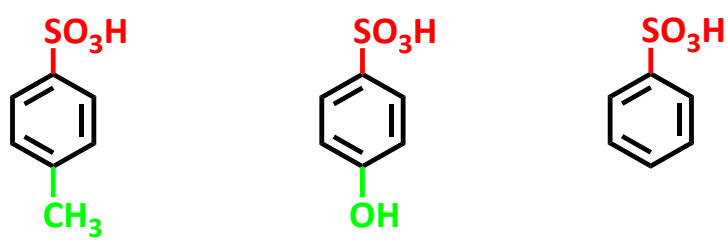

(TsOH)

(PSA)

(BSA)

Figure S17. Chemical structures of 4-Cl-BSA, di-Cl-BSA, 4-Br-BSA, TsOH, PSA, and BSA. 

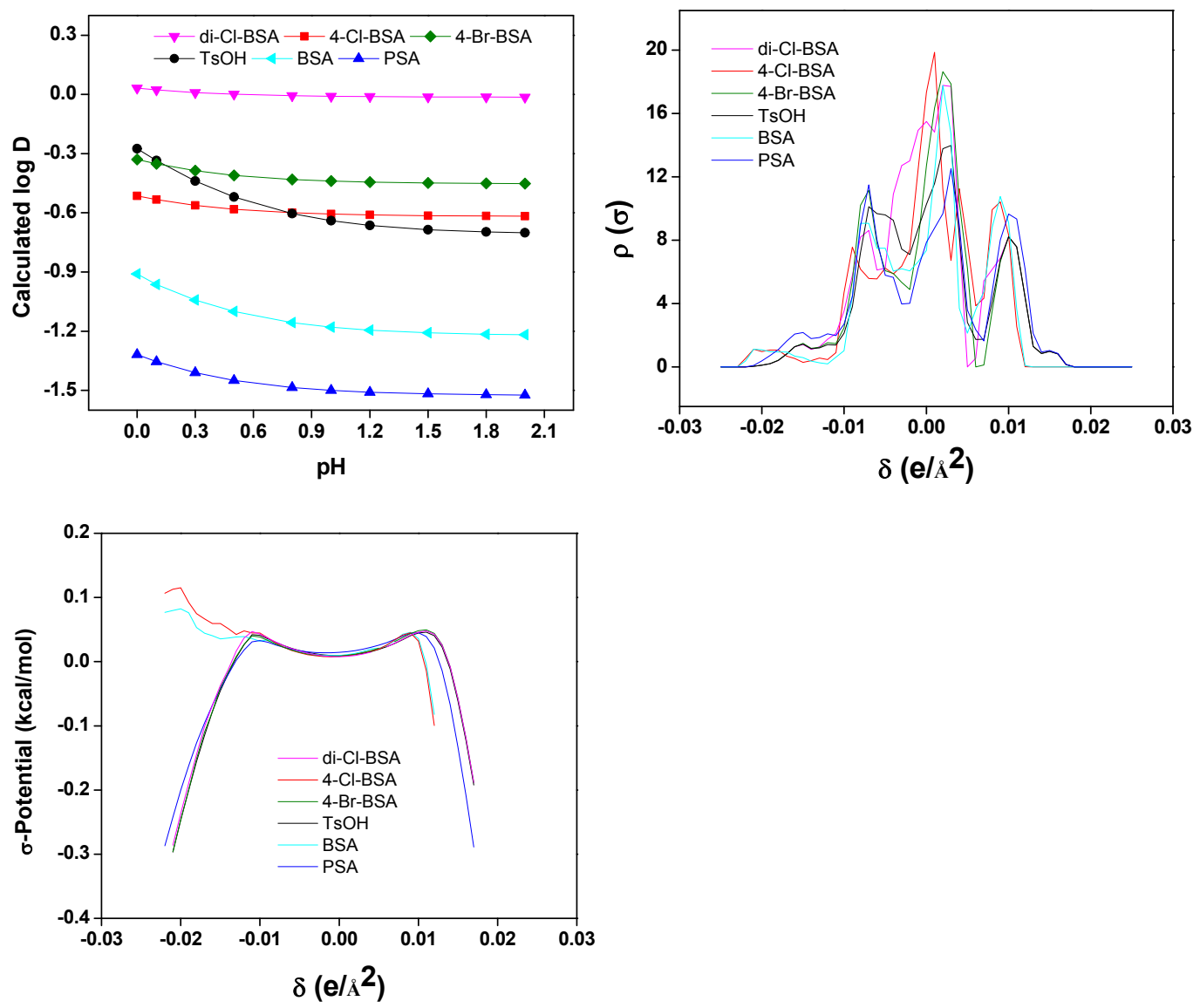

Figure S18. The calculated log D values, sigma profiles, and sigma potential profiles for di-ClBSA, 4-Cl-BSA, 4-Br-BSA, TsOH, PSA, and BSA. 
Table S1. Compositions and Inter-unit Linkage Contents of BSA-Fractionated Poplar Lignin Samples

\begin{tabular}{|c|c|c|c|}
\hline \multicolumn{2}{|c|}{ Composition (\%) } & \multirow{2}{*}{$\begin{array}{c}\text { Solubilized } \\
72\end{array}$} & \multirow{2}{*}{$\begin{array}{c}\text { Deposited } \\
60\end{array}$} \\
\hline Syringyl (S) & Total & & \\
\hline \multirow{5}{*}{ Guaiacyl (G) } & Condensed & 34 & 28 \\
\hline & Uncondensed & 38 & 32 \\
\hline & Total & 28 & 38 \\
\hline & Condensed & 22 & 23 \\
\hline & Uncondensed & 6 & 15 \\
\hline \multicolumn{2}{|c|}{ p-Hydroxyphenyl } & 0 & 0 \\
\hline \multicolumn{2}{|l|}{$\mathrm{S} / \mathrm{G}$ ratio } & 2.51 & 1.58 \\
\hline \multicolumn{2}{|c|}{ Condensed/uncondensed } & 1.26 & 1.08 \\
\hline \multicolumn{2}{|l|}{$\beta-\mathrm{O}-4$} & 6.0 & 12.0 \\
\hline \multicolumn{2}{|l|}{$\beta-\beta$} & 4.0 & 5.0 \\
\hline \multicolumn{2}{|l|}{$\beta-5$} & 2.0 & 3.0 \\
\hline
\end{tabular}


Table S2. Molecular Weights of BSA-Fractionated Poplar Lignin Samples

$\begin{array}{cccc}\text { Sample Names } & \mathrm{M}_{\mathrm{w}}(\mathrm{g} / \mathrm{mol}) & \mathrm{M}_{\mathrm{n}}(\mathrm{g} / \mathrm{mol}) & \text { PDI } \\ \text { Solubilized } & 5641 & 2061 & 2.74 \\ & 5683 & 2040 & 2.79 \\ & 6682 & 2398 & 2.79 \\ \text { Deposited } & 6690 & 2414 & 2.77\end{array}$


Table S3. The Lignin Samples from Poplar Chips Fractionated by 4-Cl-BSA and the Reported Batch and Flow-through Systems

\begin{tabular}{|c|c|c|c|c|c|c|c|c|c|c|c|c|c|}
\hline $\begin{array}{l}\text { Fractionation } \\
\text { method }\end{array}$ & Lignin & \multicolumn{2}{|c|}{$\begin{array}{l}\text { Yield } \\
(\%)\end{array}$} & $\begin{array}{l}\beta-\mathrm{O}-4 \\
\text { retention } \\
(\%)\end{array}$ & $\beta-5$ & $\beta-\beta$ & $\mathrm{S} / \mathrm{G}$ & $\begin{array}{l}\text { Condensed } \\
\text { /uncondensed }\end{array}$ & $\begin{array}{l}\mathrm{M}_{\mathrm{w}} \\
(\mathrm{g} / \mathrm{mol})\end{array}$ & $\begin{array}{l}\mathrm{M}_{\mathrm{n}} \\
(\mathrm{g} / \mathrm{mol})\end{array}$ & PDI & $\begin{array}{l}\text { Poplar } \\
\text { chip } \\
\text { size } \\
\end{array}$ & Ref \\
\hline \multirow{18}{*}{ Batch } & MWL & \multicolumn{2}{|l|}{10} & 100 & 1.42 & 9.43 & 3.08 & 0 & 11220 & 6350 & 1.77 & $\mathrm{~mm}$ & 1 \\
\hline & CEL & \multicolumn{2}{|l|}{35} & 100 & 3.8 & 5.1 & 2.31 & 0 & 13012 & 5267 & 2.47 & $\mathrm{~mm}$ & \multirow{5}{*}{$\begin{array}{l}\text { This } \\
\text { work }\end{array}$} \\
\hline & \multirow{4}{*}{$\begin{array}{l}\text { 4-Cl- } \\
\text { BSA }\end{array}$} & & dep. & 81.1 & 4.7 & 10 & 3.99 & 0.28 & 8983 & 4499 & 2.00 & \multirow{4}{*}{$\mathrm{cm}$} & \\
\hline & & & solu. & 24.7 & 2.7 & 9.9 & 2.58 & 1.07 & 10822 & 4447 & 2.43 & & \\
\hline & & \multirow[t]{2}{*}{96} & dep. & 61.9 & 3.9 & 6.0 & 3.65 & 0.16 & 6440 & 3251 & 1.98 & & \\
\hline & & & solu. & 50.7 & 5.6 & 6.0 & 4.03 & 0.22 & 7990 & 3188 & 2.51 & & \\
\hline & \multirow[t]{2}{*}{ PSA } & \multirow[t]{2}{*}{100} & dep. & 4.9 & 1.3 & 3.3 & 2.5 & 7.76 & 4024 & 1316 & 3.06 & \multirow[t]{2}{*}{$\mathrm{cm}$} & \multirow{2}{*}{$\begin{array}{l}\text { Previous } \\
\text { work }\end{array}$} \\
\hline & & & solu. & 14.7 & 2.3 & 5.8 & 3.51 & 2.32 & 5553 & 2257 & 2.46 & & \\
\hline & $\mathrm{EtOH}$ & \multicolumn{2}{|l|}{84} & 3.2 & 3 & 2 & 1.36 & 1.38 & $<2000$ & $<1000$ & $>1.5$ & \multirow{3}{*}{$\mathrm{mm}$} & \multirow{3}{*}{2} \\
\hline & THF & \multicolumn{2}{|l|}{85} & 20.6 & 2 & 2 & 1.52 & 0.45 & $<3000$ & $<1000$ & $>2.5$ & & \\
\hline & GVL & \multicolumn{2}{|l|}{52} & 53.9 & 4 & 5 & 1.79 & 0.09 & $<6000$ & $<4000$ & $>2.0$ & & \\
\hline & \multirow[t]{2}{*}{ THFA } & \multicolumn{2}{|l|}{73.1} & 48.1 & 4.6 & 8.1 & 2.45 & 0.04 & - & - & - & \multirow[t]{2}{*}{$\mathrm{mm}$} & \multirow[t]{2}{*}{3} \\
\hline & & \multicolumn{2}{|l|}{97.2} & 20.1 & 5.1 & 7.1 & 3.54 & 0.19 & - & - & - & & \\
\hline & \multirow[t]{2}{*}{ SCEP } & \multicolumn{2}{|l|}{79} & 5.9 & 3.2 & 7.7 & 1.53 & - & 6455 & 4189 & 1.54 & \multirow[t]{2}{*}{$\mathrm{cm}$} & \multirow[t]{4}{*}{4} \\
\hline & & \multicolumn{2}{|l|}{79} & 37.2 & 4.6 & 9.7 & 2.12 & - & 4485 & 2494 & 1.70 & & \\
\hline & $\mathrm{TsOH}$ & 90 & & 17.3 & 1.3 & 3.1 & 7.3 & 0.45 & 3001 & 1794 & 1.67 & $\mathrm{~mm}$ & \\
\hline & & 80 & & 7.6 & 0.9 & 2.8 & 9.7 & 0.69 & 2698 & 1679 & 1.59 & & \\
\hline & $\mathrm{FA}$ & 91.7 & & 0 & 0 & 0 & 1.5 & - & - & - & - & $\mathrm{cm}$ & 5 \\
\hline & & 41.6 & & 94.1 & 2.7 & 4.3 & 2.4 & 0.02 & - & - & - & & \\
\hline & & 20.6 & & 90.6 & 2.8 & 3.8 & 2.6 & 0.04 & 5741 & 2179 & 2.63 & $\mathrm{~mm}$ & 6 \\
\hline & TsOH & 54.2 & & 65.1 & 2.2 & 3.9 & 4.6 & 0.1 & 6807 & 2243 & 3.03 & & \\
\hline & & 76.3 & & 75.9 & 2.9 & 3.7 & 3.5 & 0.08 & - & - & - & & \\
\hline Flow- & & 50.5 & & 99.6 & 1.98 & 2.45 & 1.35 & - & - & - & - & & \\
\hline through & FA & 76 & & 91.5 & - & - & - & - & - & - & - & & \\
\hline & & 90.3 & & 83.3 & - & - & - & - & - & - & - & $\mathrm{cm}$ & 5 \\
\hline & & 94.2 & & 77.7 & - & - & - & - & - & - & - & & \\
\hline & DA & 95 & & 48.3 & 8.9 & 17.5 & & & 1083 & 1955 & 1.81 & $\mathrm{~mm}$ & 7 \\
\hline
\end{tabular}

dep.: deposited fraction; solu.: solubilized fraction; “-”: not reported; MWL: milled wood lignin; CEL: cellulolytic enzyme lignin. EtOH: the lignin isolated in $60 \%$ ethanol $/ 40 \%$ water (v/v) using $1.25 \%$ sulfuric acid (based on the poplar weight) at $180{ }^{\circ} \mathrm{C}$ for $60 \mathrm{~min}$; THF: the lignin isolated in $50 \%$ tetrahydrofuran $/ 50 \%$ water (v/v) using $0.5 \%$ sulfuric acid at $160{ }^{\circ} \mathrm{C}$ for $15 \mathrm{~min}$; GVL: the lignin isolated in $80 \%$ gamma-valerolactone $/ 20 \%$ water (v/v) using $4.41 \%$ sulfuric acid at $120{ }^{\circ} \mathrm{C}$ for $2 \mathrm{~h}$; THFA: the lignin isolated in $60 \%$ tetrahydrofurfuryl alcohol $/ 40 \%$ water $(\mathrm{v} / \mathrm{v})$ at $170{ }^{\circ} \mathrm{C}$ or $200{ }^{\circ} \mathrm{C}$ for $25 \mathrm{~min}$; SCEP: the lignin isolated in supercritical carbon dioxide $/ 50 \%$ ethanol $/ 50 \%$ water (v/v) at $120{ }^{\circ} \mathrm{C}$ for $1 \mathrm{~h}$; TsOH: the lignin isolated by the aqueous toluenesulfonic acid solution at $70-90{ }^{\circ} \mathrm{C}$ for $30-90 \mathrm{~min}$; FA: the lignin isolated in $72 \mathrm{wt} \%$ formic acid at $120-140{ }^{\circ} \mathrm{C}$ for 10-30 min; DA: the lignin isolated in water using $0.05 \%$ sulfuric acid at $240{ }^{\circ} \mathrm{C}$ for $10 \mathrm{~min}$. 


\section{References:}

(1) Chen, T.Y.; Wang, B.; Shen, X.J.; Li, H.Y.; Wu, Y.Y.; Wen, J.L.; Liu, Q.Y.; Sun, R.C. Assessment of structural characteristics of regenerated cellulolytic enzyme lignin based on a mild $\mathrm{DMSO} /[\mathrm{Emim}] \mathrm{OAc}$ dissolution system from triploid of Populus tomentosa Carr. $R S C A d v$. 2017, 7, 3376-3387.

(2) Meng, X.Z.; Bhagia, S.; Wang, Y.X.; Zhou, Y.; Pu, Y.Q.; Dunlap, J.R.; Shuai, L.; Ragauskas, A.J.; Yoo, C.G. Effects of the advanced organosolv pretreatment strategies on structural properties of woody biomass. Ind. Crops Prod. 2020, 146, 112144, 1-12. DOI:10.1016/j.indcrop.2020.112144

(3) Si, X.Q.; Lu, F.; Chen, J.Z.; Lu, R.; Huang, Q.Q.; Jiang, H.F.; Taarning, E.; Xu, J. A strategy for generating high-quality cellulose and lignin simultaneously from woody biomass. Green Chem. 2017, 19, 4849-4857.

(4) Wang, X.; Guo, Y.Z.; Zhou, J.H.; Sun, G.W. Structural changes of poplar wood lignin after supercritical pretreatment using carbon dioxide and ethanol-water as co-solvents. $R S C A d v$. 2017, 7, 8314-8322.

(5) Zhou, H.; Xu, J.Y.; Fu, Y.J.; Zhang, H.G.; Yuan, Z.W.; Qin, M.H.; Wang, Z.J. Rapid flowthrough fractionation of biomass to preserve labile aryl ether bonds in native lignin. Green Chem. 2019, 21, 4625-4632.

(6) Wang, Z.J.; Qiu, S.; Hirth, K.; Cheng, J.L.; Wen, J.L.; Li, N.; Fang, Y.M.; Pan, X.J.; Zhu, J.Y. Preserving both lignin and cellulose chemical structures: flow-through acid hydrotropic fractionation at atmospheric pressure for complete wood valorization. $A C S$ Sustainable Chem. Eng. 2019, 7, 10808-10820.

(7) Zhang, L.B.; Pu, Y.Q.; Cort, J.R.; Ragauskas, A.J.; Yang, B. Revealing the molecular structural transformation of hardwood and softwood in dilute acid flowthrough pretreatment. ACS Sustainable Chem. Eng. 2016, 4, 6618-6628. 